\title{
INSTANTONS AND NUCLEON MAGNETISM
}

\author{
H. FORKEL \\ Institut für Theoretische Physik, Universität Heidelberg, \\ D-69120 Heidelberg, Germany
}

\begin{abstract}
We construct improved QCD sum rules for the nucleon magnetic moments by implementing direct-instanton contributions to the operator product expansion of the nucleon correlator in a magnetic background field. The instanton contributions are found to affect only those sum rules which had previously been considered unstable. The new sum rules show a high degree of stability and reproduce the experimental values of the nucleon magnetic moments for values of the magnetic quark condensate susceptibility which are consistent with other estimates. (Invited talk given at "Hadron Physics 2000", Caraguatatuba, São Paulo, Brazil (April 10$15,2000)$.
\end{abstract}

Over the last years, an increasingly multifaceted picture of the role of QCD instantons 1 in hadron physics has begun to emerge from hadron models with more or less instanton-induced structure $\mathrm{B}$, instanton-liquid vacuum model $\mathbf{3}$, a generalized operator product expansion including instanton contributions (IOPE) and, most recently, lattice calculations

One of the major benefits of sharpening this picture by identifying specific hadronic instanton effects will be to end the puzzling elusiveness of explicit glue in hadrons at low energies. Indeed, in most successful models of the classical mesons and baryons, gluons play at best a marginal role. The situation is further obfuscated by the fact that gluons do not directly couple to electroweak probes. Since much of what we know about the classical hadrons derives from their response to such probes, it is particularly desirable to pin down traces of nonperturbative glue in electromagnetic hadron observables.

In this talk, I will report on an investigation 1 which takes a step towards uncovering the hidden role of glue in the response to electromagnetic fields by studying the impact of instantons on the nucleon magnetic moments in the recently developed IOPE sum-rule approach $\boldsymbol{t g}$. While in general the range of applicability of the IOPE (as a short-distance expansion) is more limited than that of instanton vacuum models or lattice calculations, it profits from the transparency of an analytical (yet largely model-independent) method and takes, in contrast to instanton models, all long-wavelength vacuum fields and also perturbative fluctuations into account.

In order to study the nucleon's magnetic moments in the framework of the IOPE, we start from a correlation function which contains the response of the nucleon to the electromagnetic current at short, spacelike distances. (The 
information on the magnetic moments is subsequently extracted by dispersion techniques 6.) At first sight, the natural candidate for such a correlation function would be

$$
\Pi_{\mu}(p, q)=-\int d^{4} x e^{i p x} \int d^{4} y e^{i q y}\left\langle 0\left|T \eta(x) J_{\mu}(y) \bar{\eta}(0)\right| 0\right\rangle
$$

where the nucleon interpolating fields $\eta(x)$ (with either proton or neutron quantum numbers) are composite operators of massless up and down quark fields $\mathrm{\theta}$,

$$
\eta_{p}(x)=\epsilon^{a b c}\left[u^{a^{T}}(x) C \gamma_{\alpha} u^{b}(x)\right] \gamma_{5} \gamma^{\alpha} d^{c}(x), \quad \eta_{n}=\eta_{p}(u \leftrightarrow d),
$$

and $J_{\mu}$ is the electromagnetic current. However, obtaining the nucleon magnetic moments requires taking the $q \rightarrow 0$ limit in which the matrix element of (11) is probed mainly in the large-distance region $|y|,|x-y| \gg \Lambda^{-1}$ where the operator product expansion breaks down $₫$.

Fortunately, there exists an alternative approach, due to Ioffe and Smilga 11 and Balitsky and Yung 2 , which can be directly applied to the calculation of the magnetic moments. The underlying idea is to treat the long-wavelength photons at the same level as the soft QCD vacuum fields (which make up the condensates), i.e. to account for them in the operators of the (I)OPE (see below). This amounts to considering the electromagnetic field as a constant, classical background field (which, incidentally, is reminiscent of the experimental setup used in actual measurements of the moments). In other words, the electromagnetic probe does not appear anymore as an external field but rather as part of the "magnetized" vacuum state.

We are thus led to consider the nucleon correlation function

$$
\begin{aligned}
\Pi(p) & =i \int d^{4} x e^{i p x}\langle 0|T \eta(x) \bar{\eta}(0)| 0\rangle_{F} \\
& =\Pi_{0}(p)+\sqrt{4 \pi \alpha} \Pi_{\mu \nu}(p) F^{\mu \nu}+O\left(F^{2}\right)
\end{aligned}
$$

in the background of a constant electromagnetic field $F_{\mu \nu}$. We have not written down higher orders of $F$ since the information on the magnetic moments is part of the linear response

$$
\Pi_{\mu \nu}(p)=\left(\not p \sigma_{\mu \nu}+\sigma_{\mu \nu} \not\right) \Pi_{1}\left(p^{2}\right)+i\left(\gamma_{\mu} p_{\nu}-\gamma_{\nu} p_{\mu}\right) \not p \Pi_{2}\left(p^{2}\right)+\sigma_{\mu \nu} \Pi_{3}\left(p^{2}\right)
$$

to the (arbitrarily weak) external field. Above we have given the general decomposition of $\Pi_{\mu \nu}(p)$ in terms of three independent Lorentz and spinor structures which are associated with one chirally-even $\left(\Pi_{1}\right)$ and two chirally-odd

$a$ The OPE of this matrix element can be used, horvever, to obtain the nucleon form factors at intermediate momentum transfers $Q \sim 1 \mathrm{GeV} 10$. 
invariant amplitudes $\left(\Pi_{2}\right.$ and $\left.\Pi_{3}\right)$. The relation to the magnetic moments can be made explicit by writing

$$
\Pi(p)=i \int d^{4} x e^{i p x}\left\langle 0\left|T \eta(x) \bar{\eta}(0) e^{-i \int d^{4} y A^{\mu} J_{\mu}}\right| 0\right\rangle
$$

where $J_{\mu}=\bar{q} \gamma_{\mu} Q q$ is the electromagnetic current with quark charge matrix $Q$ and $A_{\mu}$ is the external vector potential. In fixed-point gauge, and specializing to a constant magnetic background field

$$
B_{i}=-\frac{1}{2} \varepsilon_{i j k} F_{j k}, \quad\left(E_{i}=F_{i 0}=0\right)
$$

with the potential

$$
A_{\mu}(y)=-\frac{1}{2} F_{\mu \nu} y^{\nu},
$$

the above exponential can be rewritten as

$$
\int d^{4} y A^{\mu} J_{\mu}=\frac{1}{2} F_{i j} \int d^{4} y y_{j} J_{i}=\int d t \vec{\mu} \cdot \vec{B}
$$

where $\vec{\mu}$ is the magnetic-moment operator

$$
\vec{\mu}=\frac{1}{2} \int d^{3} x(\vec{x} \times \vec{J}) .
$$

Expanding (6) to first order in $F$ and inserting nucleon intermediate states, we thus obtain

$$
\Pi_{\mu \nu}(p) F^{\mu \nu}=-\varepsilon_{i j k} \Pi_{i j}(p) B_{k} \sim\langle N(p)|\vec{\mu}| N(p)\rangle \cdot \vec{B}
$$

in terms of the nucleon magnetic moments, as anticipated.

In order to write down sum rules for $\langle N(p)|\vec{\mu}| N(p)\rangle$, we need a QCD description of the correlator (3) at momenta $s=-p^{2} \simeq 1 \mathrm{GeV}^{2}$, i.e. at distances $x \sim 0.2 \mathrm{fm}$. Such a description is provided by the nonperturbative IOPE which factorizes (3) into contributions from soft $\left(k_{i}<\nu\right)$ and hard $\left(k_{i} \geq \nu\right)$ field modes (with momenta $k_{i}$ ), where $\nu \sim 0.5 \mathrm{GeV}$ is the operator renomalization scale. The IOPE is generated by splitting each diagram contributing to (5) in all possible ways into a hard and a soft subgraph. The hard subgraphs, with the integration range of each internal momentum restricted to be larger than $\nu$, give rise to the Wilson coefficients and receive, beyond the standard perturbative contributions 11 , direct instanton contributions which we will evaluate

${ }^{b}$ In practice, this restriction is often unnecessary. 
below. The soft subgraphs yield hadron-channel independent condensates, i.e. vacuum expectation values of local, composite QCD operators renormalized at $\nu$.

The new feature brought in by the magnetic background field is that the "magnetized" vacuum state $|0\rangle_{F}$ singles out a preferred direction (that of the field strength $\vec{B}$ ) and therefore ceases to be a Lorentz scalar. Hence, several Lorentz-covariant operators of the OPE (considered here up to dimension eight) aquire finite expectation values, the $F$-induced condensates

$$
\begin{aligned}
\left\langle 0\left|\bar{q} \sigma_{\mu \nu} q\right| 0\right\rangle_{F} & =\sqrt{4 \pi \alpha} \chi F_{\mu \nu}\langle 0|\bar{q} q| 0\rangle, \\
g\left\langle 0\left|\bar{q} G_{\mu \nu} q\right| 0\right\rangle_{F} & =\sqrt{4 \pi \alpha} \kappa F_{\mu \nu}\langle 0|\bar{q} q| 0\rangle, \\
g\left\langle 0\left|\bar{q} \gamma_{5} \tilde{G}_{\mu \nu} q\right| 0\right\rangle_{F} & =\frac{i}{2} \sqrt{4 \pi \alpha} \xi F_{\mu \nu}\langle 0|\bar{q} q| 0\rangle .
\end{aligned}
$$

$\left(G_{\mu \nu}=\frac{1}{2} \lambda_{a} G_{\mu \nu}^{a}, \tilde{G}_{\mu \nu}=\frac{1}{2} \varepsilon_{\mu \nu \rho \sigma} G_{\rho \sigma}\right.$ with $\varepsilon_{0123}=-1$.) The parameters $\chi, \kappa$, and $\xi$ quantify the vacuum response to weak electromagnetic fields and thus play the role of generalized susceptibilities. The magnetic susceptibility of the quark condensate, $\chi$, for example, originates from the induced spin alignment of quark-antiquark pairs in the vacuum. The corresponding vacuum expectation value is the lowest-dimensional induced condensate and will therefore play a leading role in the magnetic moment sum rules.

Altogether, the IOPE of the invariant amplitudes $\Pi_{i}(p)$ in Eq. (5) can thus be written as

$$
\Pi_{i}\left(p^{2}\right)=\sum_{n} C_{i, n}\left(p^{2} ; \nu\right)\left\langle 0\left|\mathcal{O}_{n}[\nu]\right| 0\right\rangle_{F}
$$

where the $\mathcal{O}_{n}$ are local, composite QCD operators with the appropriate quantum numbers (the multi-index $n$ can contain Lorentz indices) and dimension $d \leq 89$. The $C_{n}$ are the Wilson coefficients. It might be worth reiterating that the instanton contributions to (15) factorize into those from soft modes (contained in the condensates $\left\langle 0\left|\mathcal{O}_{n}\right| 0\right\rangle$ ) and those due to hard modes which add nonperturbative structure to the $C_{n}$. The latter can be of substantial size since $\bar{\rho}<\nu^{-1} \simeq 0.4 \mathrm{fm}$ and have to be calculated explicitly. (Nevertheless, they were neglected in the usual, perturbative treatment.)

The calculation of the IOPE coefficients is described in Ref. 46 to which we refer for details. Similar to the phenomenologically known values of the lowestdimensional condensates, the bulk properties of the instanton size distribution are generated by long-distance vacuum dynamics and thus taken as input. We

${ }^{c}$ Contributions from higher-dimensional operators are strongly suppressed, see below. 
will use the standard "instanton liquid" values $3 \bar{\rho} \simeq \frac{1}{3} \mathrm{fm}$ for the average instanton size and $\bar{R} \simeq 1 \mathrm{fm}$ for the average separation between neighboring (anti)instantons. Since the instanton size distribution $n(\rho)$ is rather sharply peaked around $\bar{\rho}$, we can thus approximately set $n(\rho)=\bar{n} \delta(\rho-\bar{\rho})$ with $\bar{n} \simeq 0.5$ $\mathrm{fm}^{-4}$. Moreover, $\bar{\rho}^{-1} \gg \Lambda_{Q C D}$ implies that the hard instanton contributions can be calculated semiclassically. Since, finally, multi-instanton correlations are negligible at the relevant distances $x \sim 0.2 \mathrm{fm} \ll \bar{R}$, this amounts to evaluating the hard subgraphs in the background of an (anti-) instanton field and subsequently averaging over the instanton-parameter distribution. The main contributions typically arise from the quark zero modes in the instanton field (which aquire an effective mass $13 \bar{m}(\rho)=-\frac{2}{3} \pi^{2} \rho^{2}\langle\bar{q} q\rangle$ due to the interactions with ambient vacuum fields) and can be treated exactly, while the continuum modes are approximated by plane waves.

More specifically, the leading instanton contributions to the correlator (3) can be traced to the graph in which two of the quarks propagate in zero modes while the third interacts with the background field through the magnetized quark condensate 9 . This contribution is purely nonperturbative and difficult to account for, e.g., in quark-based hadron models 15 . After averaging over the instanton size distribution, it reads

$$
\begin{aligned}
\left\langle 0\left|T \eta_{p}(x) \bar{\eta}_{p}(0)\right| 0\right\rangle_{F, \text { inst }}= & \Pi_{0}^{\text {inst }}(x)-\frac{2^{3} e_{u}}{3 \pi^{4}} \frac{\bar{\rho}^{4}}{\bar{m}^{2}} \times \\
& \left\langle\bar{q} \sigma_{\mu \nu} q\right\rangle_{F} \sigma_{\mu \nu} \int d^{4} x_{0} \frac{1}{\left(r^{2}+\bar{\rho}^{2}\right)^{3}\left(x_{0}^{2}+\bar{\rho}^{2}\right)^{3}}
\end{aligned}
$$

for the proton. $\Pi_{0}^{\text {inst }}$ contains the instanton contributions for $F=0$, calculated in 6 , and $r=x-x_{0}$, where $x_{0}$ specifies the center of the instanton. The corresponding neutron correlator is obtained by replacing $e_{u}$ with $e_{d}$. Above, we have used the self-consistency condition 16

$$
\langle\bar{q} q\rangle=-2 \int d \rho \frac{n(\rho)}{\bar{m}(\rho)}=-2 \frac{\bar{n}}{\bar{m}(\bar{\rho})}
$$

to eliminate the $\bar{n}$ dependence from (16). For later use in the sum rules, we now calculate the Fourier and Borel transform 9 of (16), from which we obtain

$$
\widehat{\Pi}_{3}\left(M^{2}\right)=\frac{e_{u}}{128 \pi^{4}} a \chi \bar{\rho}^{2} M^{6} I\left(z^{2}\right)
$$

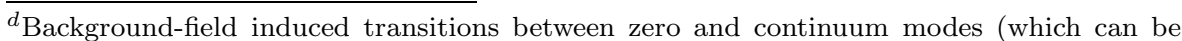
dominant in other correlators 14 ) are absent here.

$e$ For the Borel transform we follow the convention of Ref. $\mathrm{O}$. 
where $M$ denotes the Borel mass parameter and $z=M \bar{\rho}, a=-(2 \pi)^{2}\langle\bar{q} q\rangle$. The zero-mode loop gives rise to the integral

$$
I\left(z^{2}\right)=\int_{0}^{1} \frac{d \alpha}{\alpha^{2}(1-\alpha)^{2}} e^{-\frac{z^{2}}{4 \alpha(1-\alpha)}}=4 e^{-\frac{z^{2}}{2}}\left[K_{0}\left(\frac{z^{2}}{2}\right)+K_{1}\left(\frac{z^{2}}{2}\right)\right]
$$

and exhibits the unique exponential Borel-mass dependence which is characteristic for instanton contributions

A first important lesson of Eq. (18) is that, to leading order, direct instantons contribute almost exclusivelyff to the chirally-odd amplitude $\Pi_{3}$. This is crucial because in the analysis of Ref. 11, were this contribution was not accounted for, exactly the $\Pi_{3}$ sum-rule failed to show a fiducial stability region 17. The previous neglect of the instanton contributions thus offers a potential explanation for this instability. An addition Lhint in this direction comes from two other chirally-odd nucleon sum rules 15 where stabilization due to instantons was indeed found to take place?.

We are now ready to proceed to the quantitative analysis of the IOPE sum rule for $\Pi_{3}$. The latter results from equating the Borel transform of the standard OPE of Ref. 11 and the instanton contributions (18) to the Boreltransformed double dispersion relation for the correlator (3), with a spectral function parametrized in terms of the nucleon pole contribution (cf. Eq. (11)) and a continuum based on local duality. Including the infrared-divergent term encountered in Ref. 11 , duly truncated at the OPE renormalization point, the IOPE sum rule (for the proton) reads

$$
\begin{aligned}
& a M^{2}\left\{\left[e_{u}-\frac{1}{6} e_{d}(1+4 \kappa+2 \xi)\right] E_{1}(M)\right. \\
& +\frac{1}{6} e_{u} \frac{m_{0}^{2}}{M^{2}}\left[\ln \frac{M^{2}}{\nu^{2}}-\gamma_{E M}\right] L^{-\frac{4}{9}}+\frac{1}{6} e_{d} M^{2} \chi E_{2}(M) L^{-\frac{16}{27}} \\
& \left.-\frac{1}{8} e_{u} \chi \rho_{c}^{2} M^{4} I\left(z^{2}\right) L^{-\frac{16}{27}}\right\} \\
= & \frac{1}{4} \tilde{\lambda}_{N}^{2} m e^{-\frac{m^{2}}{M^{2}}}\left[\frac{\mu_{p}}{M^{2}}-\frac{\mu_{p}^{a}}{2 m^{2}}+A_{p}\right],
\end{aligned}
$$

where $m$ is the nucleon mass, $\mu_{p}\left(\mu_{p}^{a}\right)$ the proton's (anomalous) magnetic moment, $W$ the continuum threshold, and $\lambda_{N}$ the coupling of the current (2) to

$\bar{f}$ The small direct-instanton contributions to $\Pi_{2}$ have no appreciable impact on the sum rules and are neglected.

${ }^{g}$ A previously contemplated connection between the instability of the original $\Pi_{3}$ sum rule and theperturbatime infrared singularities of the "pragmatic" OPE seems, in view of newer results 18 , unlikely 6 . 
the nucleon state, $\langle 0|\eta| N\rangle=\lambda_{N} u$. For the mixed quark condensate we use the standard parametrization $\left\langle\bar{q} \sigma_{\mu \nu} G^{\mu \nu} q\right\rangle=-m_{0}^{2}\langle\bar{q} q\rangle$ with $m_{0}^{2}=0.8 \mathrm{GeV}^{2}$, and $\gamma_{E M} \simeq 0.577$ is the Euler-Mascheroni constant. The additional parameters $A_{p, n}$ determine the strength of electromagnetically induced transitions between the nucleon and its excited states. The sum rule for the neutron is obtained from (20) by interchanging $e_{u}$ and $e_{d}$ and by replacing $\mu_{p}, \mu_{p}^{a} \rightarrow \mu_{n}$ and $A_{p} \rightarrow A_{n}$. We have also defined $\tilde{\lambda}_{N}^{2}=32 \pi^{4} \lambda_{N}^{2}, L=\ln (M / \Lambda) / \ln (\mu / \Lambda)$ $(\Lambda=0.1 \mathrm{GeV})$, and transferred, using the standard expressions

$$
E_{n}(M)=1-e^{-\frac{W^{2}}{M^{2}}}\left[1+\sum_{1}^{n} \frac{1}{j}\left(\frac{W^{2}}{M^{2}}\right)^{j}\right],
$$

the continuum contributions to the IOPE-side of the sum rules. The appropriate form of these contributions has recently been clarified in Ref. 19 .

The quantitative analysis of the IOPE sum rule (20) is based on the minimization of the relative deviation between its two sides. Since the fiducial domain, i.e. the Borel-mass region in which the IOPE truncation is justified while the nucleon pole still dominates over the continuum, is (as usual) not large enough to determine all unknown parameters from a combined fit, we follow the procedure of Ref. 11 and obtain the coupling $\tilde{\lambda}^{2}=2.93 \mathrm{GeV}^{6}$ and the continuum threshold $W=1.66 \mathrm{GeV}$ by fitting the instanton-improved nucleon mass sum rule of Ref. 7 to the experimental nucleon mass. The values of the two susceptibilities $\kappa=-0.34 \pm 0.1$ and $\xi=-0.74 \pm 0.2$ were estimated in independent work by Kogan and Wyler 20. This enables us to fit both sides of the sum rules (20) by varying $\chi$ and $A_{p}$ (or $A_{n}$, respectively) while keeping the magnetic moments fixed at their experimental values.

The fits are performed in the fiducial Borel mass domain $0.8 \mathrm{GeV} \leq M \leq$ $1.15 \mathrm{GeV}$, where the highest-dimensional operators contribute at most $10 \%$ to the IOPE and the continuum contribution does not exceed $50 \%$. This fiducial domain is larger, incidentally, than that of the sum rules based on $\Pi_{1}$ and $\Pi_{2}$.

Figure 1 shows, both for the proton and the neutron sum rules, the directinstanton contributions, the remaining OPE including the continuum contributions, their sum (which makes up the left-hand side of Eq. (20)), and the right-hand sides. The fit quality of both the proton and neutron sum rules is quite impressive. Figure 1 shows, furthermore, that the direct-instanton contributions can reach the magnitude of the remaining terms in the OPE, which explains why their previous neglect had a detrimental impact on the sum-rule stability. In Fig. 2 the optimized sum rules are solved for $\mu_{N}$ and plotted as a function of the Borel mass. The resulting functions $\mu_{p, n}(M)$ therefore specify the value of the magnetic moment which is required to make both sides of the 


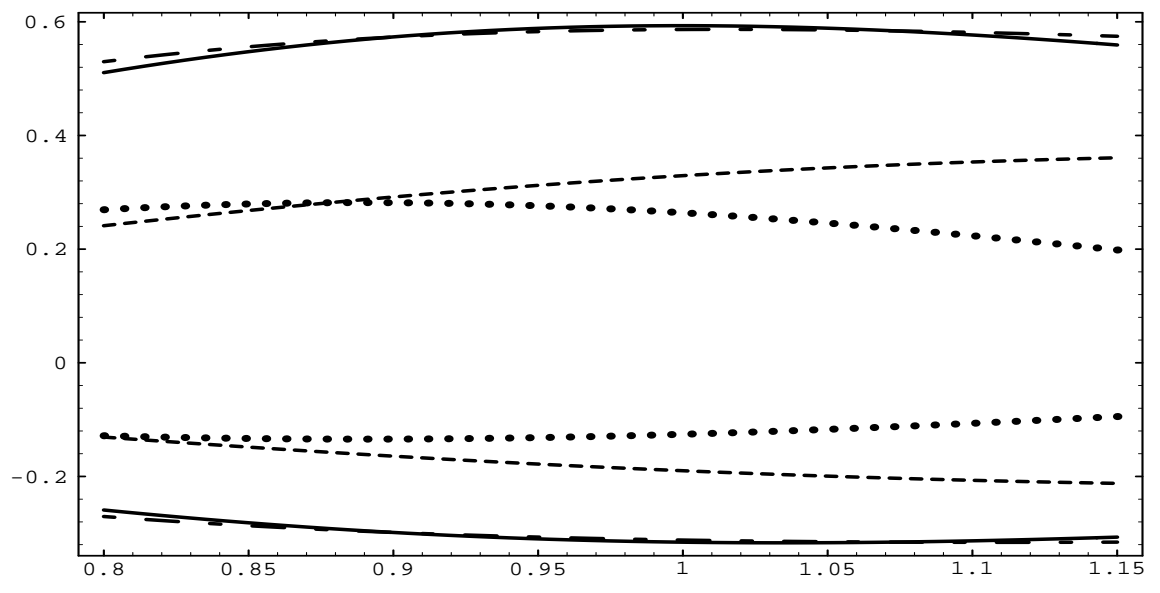

Figure 1: The OPE (dashed line) and direct instanton (dotted line) contributions to the new $\sigma_{\mu \nu}$ sum rules for the proton (positive range) and neutron. Their sum (dot-dashed line) is compared to the RHS (solid line).

sum rule (20) match exactly at a given value of $M$. As a consequence of the high fit quality, $\mu(M)$ is practically $M$-independent. The numerical results are $\chi \simeq-4.96 \mathrm{GeV}^{-2}, A_{p} \simeq 0.28 \mathrm{GeV}^{2}$ for the proton and $\chi \simeq-4.73 \mathrm{GeV}^{-2}$, $A_{n} \simeq-0.27 \mathrm{GeV}^{-2}$ for the neutron sum rule. The values of the quark condensate susceptibility $\chi$ (at $\nu=0.5 \mathrm{GeV}$ ) lie within the range obtained from other estimates 11.18 .21 and differ somewhat from the value $\chi \simeq-5.7 \mathrm{GeV}^{-2}$ found in the two- and three-pole models of Ref. 21.

In conclusion, we have recovered a third reliable sum rule for the nucleon magnetic moments. In contrast to the other two, it receives previously neglected direct-instanton contributions which arise from the interplay with long-wavelength vacuum fields. Our new sum rule is built on the chirally-odd amplitude $\Pi_{3}$ of the nucleon correlator in an electromagnetic background field and found to be at least as stable as the other two (although it had previously been regarded as flawed). The new sum rule adds to the predictive power of the background-field sum rules and strengthens their mutual consistency.

Moreover, our results reinforce a systematic pattern which_emerged from the study of direct-instanton effects in the pion 14 , nucleon 15 and glueball 22 channels: those sum rules which worked satisfactorily without instanton corrections receive little or no direct instanton contributions while previously 


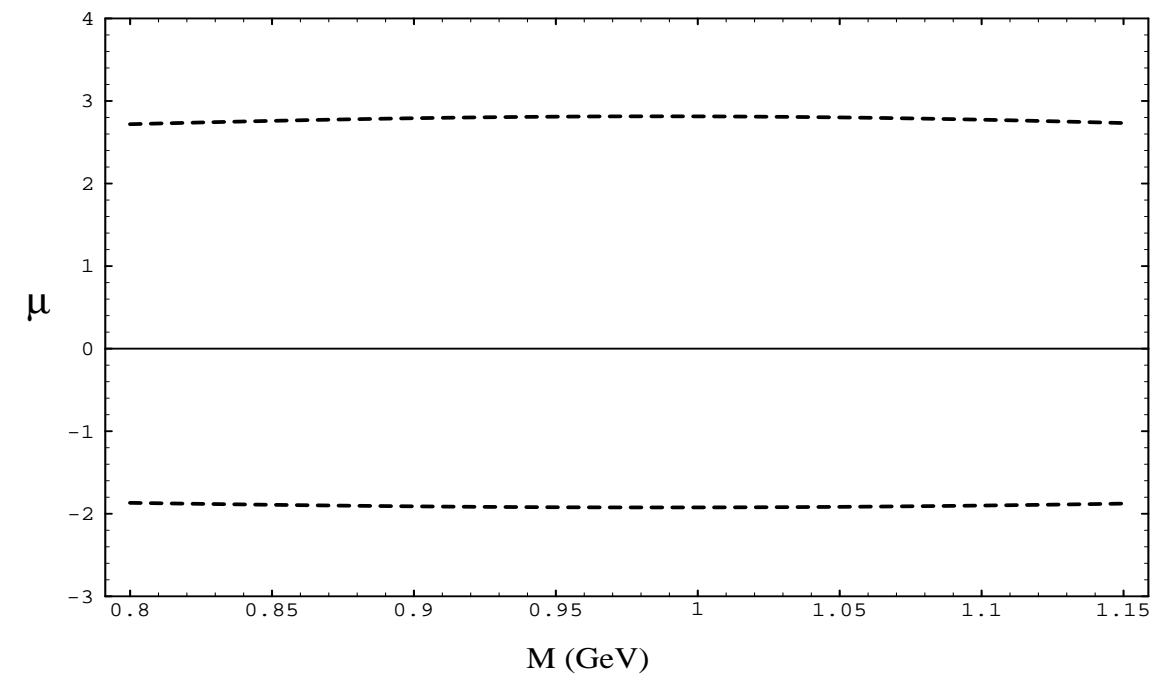

Figure 2: The Borel mass dependence of the magnetic moments of the proton (upper) and neutron calculated from the optimal fit of LHS and RHS.

less reliable or completely unstable sum rules are stabilized by large instanton contributions. This pattern points not only towards the importance of direct instantons in particular sum rules, but also supports the adequacy of their semiclassical implementation into the OPE. Our results show that these conclusions continue to hold in the presence of a "magnetized" vacuum.

\section{Acknowledgments}

I would like to thank the organizers for this interesting and stimulating meeting, and for choosing such a beautiful location. I would also like to thank Mountaga Aw and Manoj Banerjee for their collaboration on the work presented here, which was supported by the Deutsche Forschungsgemeinschaft under habilitation grant Fo 156/2-1 and by the U.S. Dept. of Energy under grant number DE-FG02-93ER-40762.

\section{References}

1. A.A. Belavin, A.M. Polyakov, A.S. Schwartz and Yu.S.Tyupkin, Phys. Lett. B 59, 85 (1975); R. Jackiw and C. Rebbi, Phys. Rev. Lett. 37, 172 (1976); C.G. Callan Jr., R.F. Dashen and D.J. Gross, Phys. Lett. B 
63, 334 (1976); G. 't Hooft, Phys. Rev. Lett. 37, 8 (1976).

2. D. Horn and S. Yankielowicz, Phys. Lett. B 76, 343 (1978); W.U. Blask, U. Bohn, M. Huber, B. Metsch, and H. Petry, Z. Phys. A 337, 327 (1990); A.E. Dorokhov, Y.A. Zubov, and N.I. Kochelev, Sov. J. Part. Nucl. 23, 522 (1992); A. Christov et al., Prog. Part. Nucl. Phys. 37, 91 (1996).

3. T. Schäfer and E. Shuryak, Rev. Mod. Phys. 70, 323 (1998); D.I. Diakonov and V.Yu. Petrov, Nucl. Phys. B245, 259 (1984); Phys. Lett. B 147, 351 (1984); Nucl. Phys. B272, 457 (1986).

4. H. Forkel, Instantons, OPE and Hadron Structure, to be published.

5. M.C. Chu and S. Huang, Phys. Rev. D 45, 2446 (1992); M.-C. Chu, J.M. Grandy, S. Huang and J. Negele, Phys. Rev. D 49, 6039 (1993); P. van Baal, Nucl. Phys. Proc. Suppl. 63, 126 (1998).

6. M. Aw, M.K. Banerjee and H. Forkel, Phys. Lett. B 454, 147 (1999).

7. H. Forkel and M.K. Banerjee, Phys. Rev. Lett. 71, 484 (1993).

8. M.A. Shifman, A.I. Vainshtein and V.I. Zakharov, Nucl. Phys. B147, 385,448 (1979).

9. B.L. Ioffe, Nucl. Phys. B188, 317 (1981), Nucl. Phys. B191, 591(1981); V.M. Belyaev and B.L. Ioffe, Sov. Phys. JETP 56, 493 (1982).

10. V.M. Belyaev and I.I. Kogan, Int. J. Mod. Phys. A 8, 153 (1993).

11. B.L. Ioffe and A.V. Smilga, Nucl. Phys. B232, 109 (1984).

12. I.I. Balitsky, Phys. Lett. B 114, 53 (1982); I.I. Balitsky and A.V. Yung, Phys. Lett. B 129, 328 (1983).

13. M.A. Shifman, A.I. Vainshtein and V.I. Zakharov, Nucl. Phys. B163, $46(1980)$

14. H. Forkel and M. Nielsen, Phys. Lett. B 345, 55 (1995).

15. H. Forkel and M. Nielsen, Phys. Rev. D 55, 1471 (1997).

16. C.G. Callen Jr., R. Dashen and D.J. Gross, Phys. Rev. D 17, 2717, 2763, (1978); D.G. Caldi, Phys. Rev. Lett. 39, 121 (1977).

17. B. Ioffe and A. Smilga, private communication.

18. S.L. Wilson, J. Pasupathy and C.B. Chiu, Phys. Rev. D 36 , 1451 (1987); C.B. Chiu, S.L. Wilson, J. Pasupathy and J.P. Singh, Phys. Rev. D 36, 1553 (1987).

19. B. Ioffe, Phys. Atom. Nucl. 58, 1408 (1995).

20. I.I. Kogan and D. Wyler, Phys. Lett. B 274, 100 (1992).

21. V.M. Belyaev and Ya.I. Kogan, Yad. Fiz. 40, 1035 (1984) [Sov. J. Nucl. Phys. 40, 659 (1984)]; Ya. Balitsky, A.V. Kolesnichenko, and A.V. Yung, Yad. Fiz. 41, 282 (1985) [Sov. J. Nucl. Phys. 41, 178 (1985)].

22. H. Forkel, Heidelberg preprint HD-TVP-00-1 (2000), hep-ph/0005004. 